\title{
Querer No Basta: Deberes Éticos en la Práctica, Formación e Investigación en Psicología Comunitaria
}

\author{
To Want is Not Enough: Ethical Duties in Practice, \\ Training, and Research in Community Psychology
}

\author{
María Inés Winkler \\ Universidad de Santiago de Chile
}

\author{
Katherine Alvear y Bárbara Olivares \\ Universidad Diego Portales
}

\author{
Diana Pasmanik \\ Universidad de Santiago de Chile
}

\begin{abstract}
Se expone una síntesis de los resultados del proyecto de investigación FONDECYT N 1080528 , en el que se indagó la dimensión ética en la práctica, formación e investigación en psicología comunitaria (PC) en Chile. Se utilizó una muestra opinática e intencionada de fuentes primarias (93 participantes —usuarios/as, estudiantes y psicólogos/as-) y fuentes secundarias de información (194 documentos - programas de asignaturas, tesis y códigos-). Del empleo de diversas técnicas de recolección de datos y estrategias cualitativas de análisis emerge como resultado relevante el principio de respeto por el otro y la participación de la comunidad para la identificación y enfrentamiento de problemas éticos. Asimismo, se hallaron divergencias respecto del concepto de PC y de los deberes éticos en este campo. Finalmente, se sugieren orientaciones éticas para la práctica, formación e investigación en PC.
\end{abstract}

Palabras clave: ética, práctica, formación, investigación, psicología comunitaria

\begin{abstract}
The article presents a summary of the results of the research project FONDECYT $\mathrm{N}^{\circ} 1080528$, which explores the ethical dimension in practice, training, and research in community psychology (CP) in Chile. An intentional opinatic sample of primary (93 participants-users, students, and psychologists-) and secondary information sources (194 documents-programs, courses, theses and codes-) was used. The use of various data collection techniques and qualitative analysis strategies led to the identification of two relevant results: the principle of respect for the other and community participation in identifying and addressing ethical issues. Divergences in the concept of CP and ethical duties in this field were also found. Finally, ethical guidelines for practice, training, and research in $\mathrm{CP}$ are suggested.
\end{abstract}

Keywords: ethics, practice, training, research, community psychology

\section{Valores y Principios Éticos en Psicología Comunitaria (PC): Consensos y Nuevos Desafíos}

La PC, con su larga trayectoria en Latinoamérica, emerge como una práctica que reacciona ante la crisis de legitimidad y significancia social que aquejaba a la psicología social (Montero, 2004a; Serrano-García \& Vargas, 1993), mientras en Estados Unidos y Europa surge como un cuestionamiento a la psicología clínica tradicional y los problemas de accesibilidad a los servi-

María Inés Winkler, Escuela de Psicología, Universidad de Santiago de Chile; Katherine Alvear y Bárbara Olivares, Facultad de Psicología, Universidad Diego Portales, Santiago, Chile; Diana Pasmanik, Escuela de Psicología, Universidad de Santiago de Chile.

Esta investigación cuenta con el patrocinio de la Universidad de Santiago de Chile y ha sido financiada por el Fondo Nacional de Desarrollo Científico y Tecnológico (FONDECYT), Proyecto No 1080528, titulado Cuestiones Éticas en la Práctica, Formación e Investigación en Psicología Comunitaria.

La correspondencia relativa a este artículo debe ser dirigida a María Inés Winkler, Escuela de Psicología, Universidad de Santiago de Chile, Avda. Ecuador 3650, $3^{\text {er }}$ piso, Estación Central, Santiago, Chile. E-mail: maria. winkler@usach.cl 
cios psicológicos por parte de grandes grupos poblacionales (Hombrados, 1996). Esta distinción reafirma la importancia de los contextos histórico-culturales en la generación de ciencia y profesión (Cushman, 1993), como una práctica que enfatiza la unión de teoría y acción en función de la realidad social específica en la cual se aplica, aunque existe controversia respecto de cuánto de ello ha sido logrado (Wiesenfeld, 2000).

Los actuales desarrollos de la $\mathrm{PC}$ en el mundo derivaron de tales trayectorias disímiles en cada hemisferio. El sello clínico de la PC anglosajona deriva de las reflexiones en torno al Movimiento de Salud Mental Comunitaria, que promovía la tendencia desinstitucionalizadora en el tratamiento de las enfermedades mentales, para superar la sobrecarga de los lugares de tratamiento de enfermos y su conversión en "depósitos" de seres humanos. Asimismo, la insatisfacción creciente con la psicoterapia como único modo de intervención psicológica y la necesidad de considerar el contexto impulsaron tales reflexiones. En Latinoamérica, en tanto, la PC se caracteriza por definirse más como una práctica que como una nueva rama de la psicología (Montero, 2004b), buscando estrategias para contribuir, no solo a estudiar, sino, principalmente, a aportar soluciones a los problemas psicosociales urgentes, con un marcado acento en la transformación social. Así, la PC anglosajona inaugura un nuevo quehacer psicológico que responde a exigencias de legitimidad social y transformación de las instituciones, mientras la PC latinoamericana se enfoca en la generación de una praxis orientada hacia la solución de problemas sociales, con la participación de las personas involucradas, lo que supone considerar nuevos actores sociales y un nuevo rol para los/as psicólogos/as ${ }^{1}$ (Montero, 2004b).

Por otra parte, los cambios que han caracterizado a la PC en Chile han sido ampliamente documentados en los últimos años (Alfaro, 2007; Alfaro \& Zambrano, 2009; Asún, 2007; Krause, 2002; Krause \& Jaramillo, 1998). Una creciente institucionalización, dada por la implementación de objetivos y métodos comunitarios en el desarrollo de programas estatales, ha ampliado el campo de inserción profesional para los psicólogos de nuestro país.

Pensamos que, como consecuencia de tal institucionalización y creación de nuevos programas sociales —actualmente 360 en el país (Jimeno, 2011, Abril 11)—, emergen nuevos desafíos profesionales, tanto técnicos como éticos, para quienes se desempeñan en este ámbito. Ante este nuevo panorama profesional nos preguntamos, específicamente, por las cuestiones éticas en la práctica, formación e investigación en PC.

La ética ha sido definida como una disciplina filosófica que reflexiona sobre las costumbres, a través de los usos de principios y normas, para responder acerca de cómo debe vivirse la vida (Orellana, 1994). Bolívar (2005) la considera una reflexión crítica de segundo orden acerca de la experiencia moral humana, prescribiendo los comportamientos. La moral se refiere a los hábitos y costumbres que regulan la propia actuación y la convivencia social (Montero, 2004a) y las profesiones establecen sus normas, a través de un consenso entre pares y a partir de los principios éticos universales, en los códigos deontológicos que contienen la "voz de la conciencia" profesional (Bersoff, 1995, p. 1).

Aprender ética y el código deontológico promulgado por los psicólogos corresponde a uno de los componentes principales y esenciales de la socialización de los estudiantes en la profesión, ya que enseña cómo un grupo de asociados define sus responsabilidades hacia un otro y el resto de la sociedad (Erikson, 1967).

Los principios éticos poseen un carácter aspiracional, en tanto las normas éticas de los códigos se refieren al deber ser en el quehacer profesional; en la práctica profesional se espera que ambos se manifiesten como un querer actuar de acuerdo a ellos.

\footnotetext{
1 Las autoras asumen la importancia de la distinción lingüística de género; sin embargo, para facilitar la lectura del texto, en adelante esta será obviada.
} 
Los principales desarrollos en los aspectos éticos profesionales en psicología se remiten a la psicología clínica; la PC plantea nuevas problemáticas que no se resuelven aplicando la deontología clínica, lo que es válido también para otras áreas de la psicología (e.g., jurídica y forense, educacional). De hecho, investigaciones previas han reportado que las representaciones acerca de la ética en el ejercicio profesional de la psicología en Chile siguen fuertemente asociadas al rol clínico (Reyes, 2009; Winkler \& Reyes, 2006), lo que parece concordante con la mayor tradición de la psicología clínica — versus la menor trayectoria de la PC-y un consecuente desarrollo de una normativa deontológica que encuadra y orienta su quehacer.

Diversos autores postulan que la PC promueve valores específicos y enfrenta temas y problemas éticos propios y característicos de sus desarrollos teóricos y prácticas profesionales (Montero, 2004b; Prilleltensky, 2001; Sánchez Vidal, 1999). Esto contrasta con los nuevos ámbitos de aplicación, roles profesionales y prácticas tanto en Chile (Alfaro, 2007) como en Estados Unidos (Jenkins, 2010) y España (Berdullas, Santolaya \& Fernández, 2002), sin acompañarse de una normativa deontológica específica (Winkler, 2007) que se constituya en una guía y orientación para el quehacer profesional. Tal ausencia es aún mayor en la investigación y la formación en PC.

El lugar de la ética como una preocupación que se sitúa por sobre la hegemonía de la técnica ha estado presente en la PC junto a otros ámbitos disciplinares, como la psicología crítica y la psicología de la liberación (ambas de origen latinoamericano), que han expresado explícitamente los aspectos valóricos que inspiraron, desde sus inicios, su quehacer. Por ejemplo, Martín-Baró consideraba que los psicólogos deben tener un compromiso crítico con las personas con las que trabajan. Asimismo, estas perspectivas destacan transversalmente la reflexividad como condición necesaria para el trabajo, entendiéndola como la capacidad de examinar constantemente el quehacer, de abrir procesos de reflexión, compartidos con todos como colaboradores, coautores del proceso de producción de conocimiento (Montero, 2004b).

Específicamente en la PC, Marconi (1986) promovió el respeto a la cultura popular, Rappaport (1976) señaló la importancia de poseer un sesgo multicultural y el derecho a la diferencia, Heller (1989) y Prilleltensky (2001) relevaron la búsqueda de justicia y equidad social, Levine y Perkins (1997) y Montero (1984) han destacado la importancia de la potenciación o empowerment de los más débiles y Sánchez Vidal (1999) ha enfatizado la participación y la creencia en que las comunidades tienen sus recursos o potencialidades para identificar problemas y resolverlos. Destaca, en este contexto, la propuesta de Prilleltensky (2001) de una práctica comunitaria basada en valores, en especial, la justicia social, para lo cual propone orientaciones para hacer coherentes los valores de la PC, sus prácticas y las políticas públicas.

Asimismo, los conceptos teóricos de participación y empowerment (Rappaport, 1981; Rappaport, Swift \& Hess, 1984; Silva \& Martínez, 2007) y su alta valoración implican, no solo respetar profundamente al otro, sino también promover el poder en el otro. Lo comunitario incluye el rol activo de la comunidad y la garantía de su participación, no solo como invitada o como espectadora o receptora de beneficios, sino como agente activo con voz, voto y veto. Asimismo, la PC acentúa el desarrollo de las fortalezas y capacidades de las comunidades por sobre sus debilidades y carencias. Los psicólogos son, entonces, agentes de transformación social que comparten su conocimiento con otros actores sociales, provenientes de la comunidad, poseedores de un saber y orientados por los mismos objetivos, con los cuales trabajan conjuntamente (Montero, 2004b).

La PC, al buscar una relación más igualitaria, procurando redistribuir el poder y conocimiento, tradicionalmente concentrado en instituciones y especialistas, a los miembros de la comunidad, disminuye - aunque no elimina - el riesgo de transgresiones éticas. Por el contrario, el encuadre tradicional en psicología clínica conlleva el establecimiento de una relación de mayor desigualdad — alguien ayuda y alguien es ayudado—, condición para la ayuda efectiva y terreno fértil para las trasgresiones a través del abuso de poder (Pope, 1990, 1991; Winkler, 
1999). Sin embargo, también se corre el riesgo de suponer que, porque en el trabajo comunitario se comparte - teóricamente- un conjunto de valores, no existe el peligro constante de cometer faltas éticas. Es así como ya O’Neill (1989) reconoce el desinterés y falta de diligencia del campo comunitario para confrontar los temas éticos, mientras Davidson (1989, citado en Sánchez Vidal, 1999) los atribuye a una "identificación angélica" que hace que el interventor comunitario se sitúe autoindulgentemente por encima del bien y el mal y que podríamos expresar como: nosotros somos los "buenos", tenemos los valores correctos, y los demás "malos" o hipócritas, fingen neutralidad cuando en realidad están sosteniendo los valores dominantes y apuntalando el statu quo. Esta postura "angelical" asociada a la aceptación casi incondicional de los valores del otro - a propósito del relativismo cultural- dejaría a los interventores en una situación de vulnerabilidad, al otorgarles un rol de expertos invitados con escaso control sobre las acciones y explicaría la "persistente omisión de temas éticos en la intervención social" (Sánchez Vidal, 1999, p. 179).

Así, también, el meta-análisis sobre publicaciones en revistas chilenas de psicología entre 1992 y 2003 que realiza Reyes (2007) muestra que "en general, las orientaciones ético-valóricas de la PC nacional de los últimos años se traducen en acciones que, desde un polo negativo, carencial y deficitario, relegan a un plano secundario la consideración de aspectos promocionales y transformadores en la comunidad" (p. 134). Por lo tanto, la explicitación valórica de la PC no garantiza su aplicación al momento de la práctica misma.

A partir de los antecedentes presentados, que denuncian un contexto de alta exigencia en desafíos éticos y escaso marco normativo que oriente y guíe el quehacer profesional, elaboramos un proyecto de investigación con el objetivo de identificar y describir las temáticas éticas propias de la práctica, formación e investigación en PC, para concluir formulando sugerencias éticas específicas para disminuir la brecha existente entre los principios aspiracionales que la fundan y la realidad de su quehacer en Chile.

\section{Método}

Para este estudio empleamos un diseño exploratorio-descriptivo, siguiendo una lógica constructivo-interpretativa (González, 1998). Para asegurar calidad y profundidad en la investigación combinamos diferentes estrategias y fuentes de información (Flick, 1990; Martínez, 1999). Privilegiamos los métodos cualitativos, por sus ventajas epistemológicas y metodológicas para la aproximación a realidades sociales complejas (Álvarez, 2000; González, 2002), así como por su concordancia con la PC, respecto de la valoración de la diversidad, del contexto y la importancia de los sujetos sociales (Banyard \& Miller, 1998; Stein \& Mankowski, 2004). Articulamos las estrategias metodológicas en un sistema de módulos temáticos complementarios, que presentamos en las Tablas 1 y 2, organizados según las fuentes de información utilizadas.

El tipo de muestreo que empleamos fue opinático e intencionado (Polit \& Hungler, 2002), pues consistió en la selección según criterios preestablecidos de documentos (194) e informantes (93 personas participaron voluntariamente, según acreditamos en los respectivos consentimientos informados).

Organizamos los resultados en los tres ámbitos de interés: práctica (fueron las fuentes de información, principalmente los módulos 2, 3, 4, 5, 6 y 8), formación (módulos 1, 2, 4, 5 y 6) e investigación en PC (módulos 2, 4, 6, 7 y 8). 


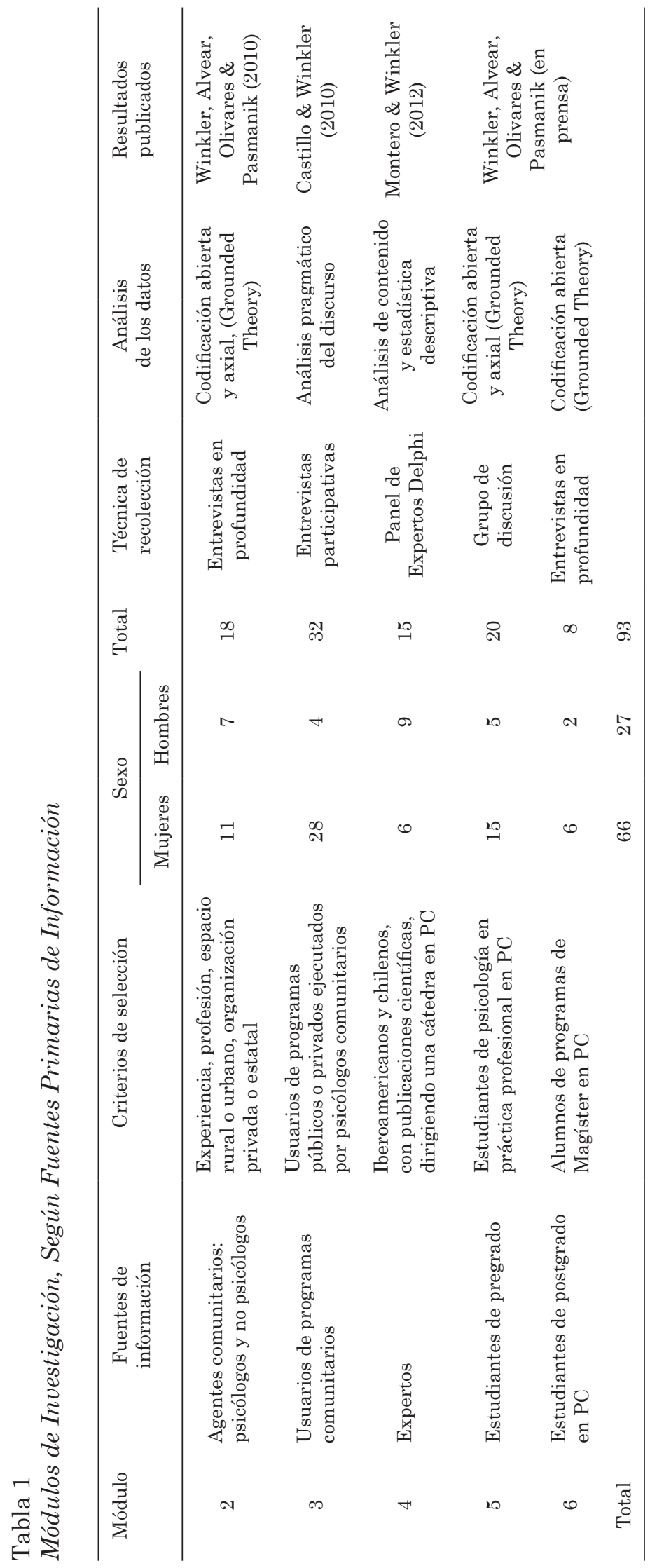




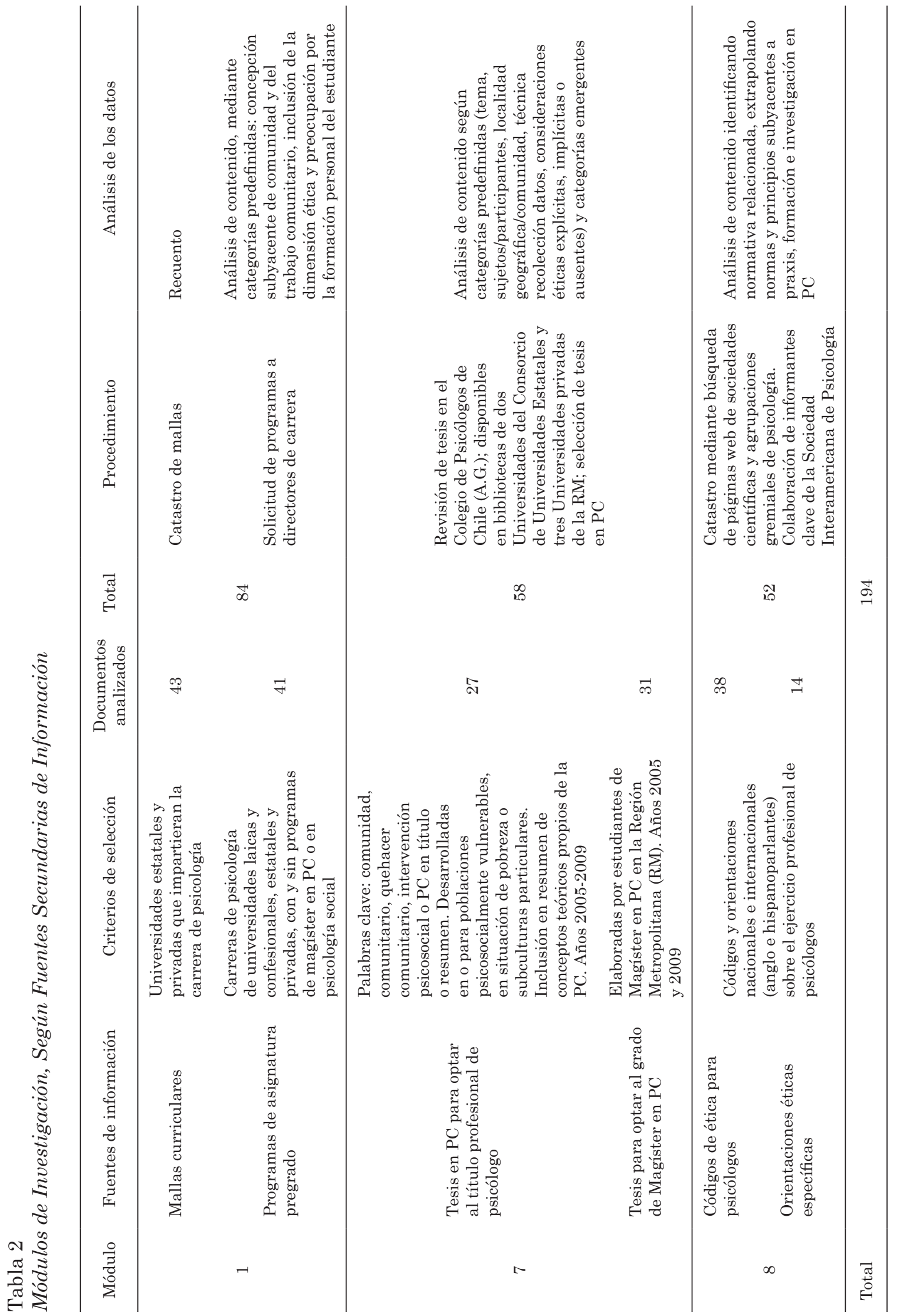




\section{Resultados}

Presentamos los resultados generales de la investigación divididos en dos apartados. En el primero describimos los principales problemas éticos para los ámbitos de la práctica, formación e investigación en PC, recabados del análisis de las fuentes primarias y secundarias, mostrando una síntesis, pues, tal como se indica en la Tabla 1, los resultados en profundidad son reportados en publicaciones específicas.

En el segundo apartado presentamos una propuesta de orientaciones éticas para el ejercicio en cada una de las áreas de aplicación mencionadas.

\section{Cuestiones Éticas en la Práctica, Formación e Investigación en PC}

En este apartado reunimos los principales problemas éticos para las tres áreas de nuestra investigación y, en términos globales, son ilustrados en la Figura 1.

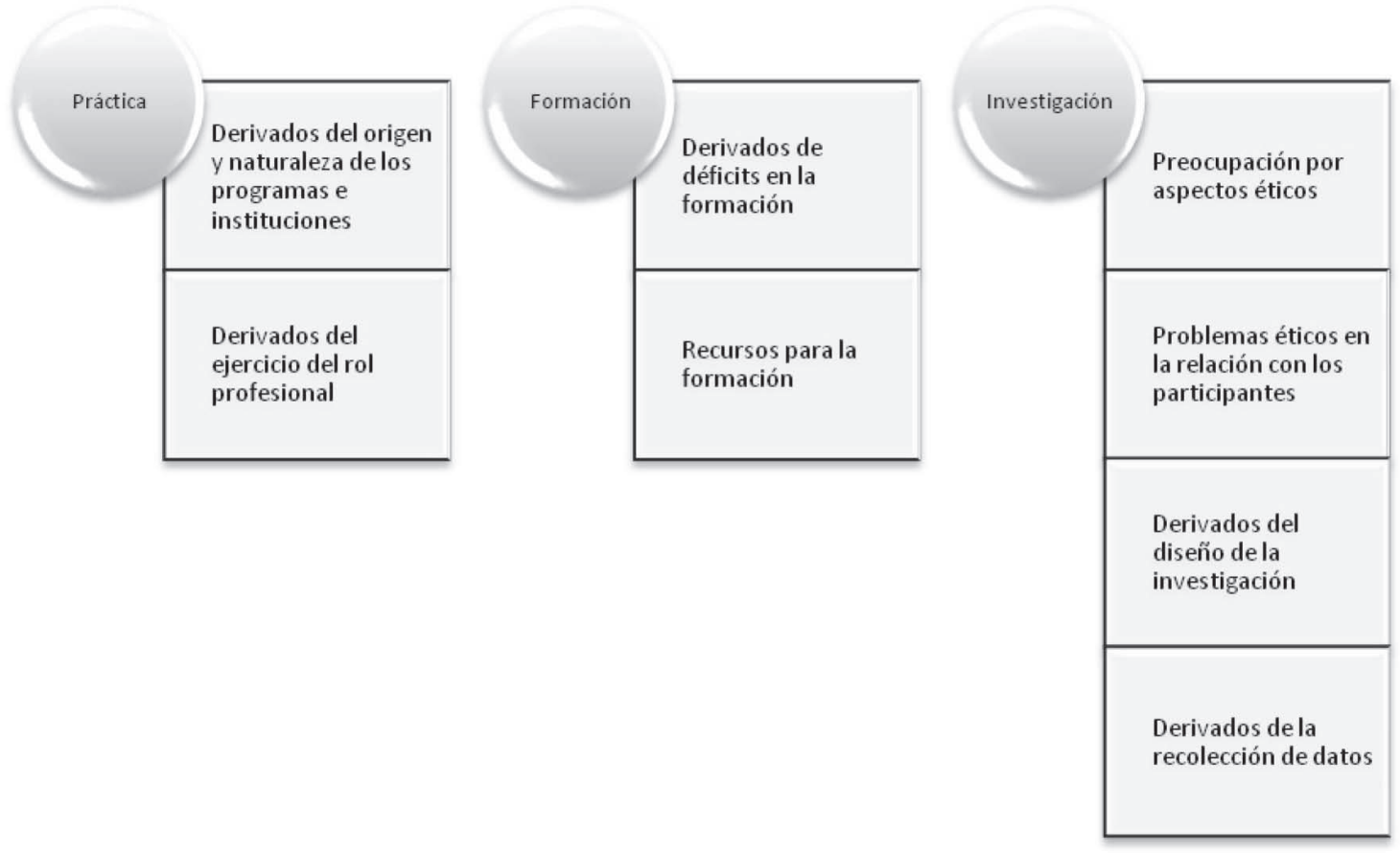

Figura 1. Categorías descriptivas de resultados globales. Temas éticos en PC.

Temas éticos en la práctica. Corresponde al ámbito más saturado y se refiere principalmente a las problemáticas mencionadas por usuarios, estudiantes y expertos del panel Delphi, pero, sobre todo, por psicólogos y no psicólogos que se desempeñan en los espacios comunitarios.

En primer lugar, los participantes describen problemas que derivan del origen y la naturaleza de los programas o instituciones en que el psicólogo desarrolla sus intervenciones, con financiamiento predominantemente estatal. La dependencia política y de lógicas electorales pone en riesgo la continuidad y eficacia de las intervenciones y, por lo tanto, el bienestar de los usuarios. En ocasiones, los psicólogos enfrentan contextos institucionales que no permiten proyectar las intervenciones más allá de cuatro años, tiempo equivalente al período de elecciones 
en Chile, tanto municipales como presidenciales. Los entrevistados reportan que, luego de un cambio de autoridad local, han dejado de existir programas y se han interrumpido dramáticamente intervenciones, lo que conlleva una preocupación de los profesionales por el efecto en el otro y en la superación de su problemática. Esto, además, genera pérdidas económicas, al suspender programas que luego son reinstalados, invirtiendo fondos públicos sin la necesaria evaluación de su efectividad e impacto.

Otra consecuencia, según los entrevistados, es la modificación de los objetivos de las intervenciones en función del cambio programático del gobierno local de turno. Otro efecto negativo es la supeditación del financiamiento al cumplimiento de ciertos indicadores cuantitativos arbitrarios, que suelen no ser consistentes con los objetivos de las intervenciones propuestas.

Los participantes refieren, además, el desarrollo de intervenciones comunitarias con diseños inadecuados, que se proponen metas ambiciosas en contraste con los plazos de ejecución; por ejemplo, proyectos para el fortalecimiento de la identidad comunitaria que cuentan con un financiamiento de solo seis meses, sin considerar los tiempos requeridos para un adecuado proceso de familiarización, que permita incorporar apropiadamente a la comunidad, respetando sus prioridades o necesidades. En este sentido, aparece la preocupación por la escasa - o ausente- participación de la comunidad en la formulación de los programas de intervención. Los psicólogos entrevistados develan la prevalencia de una lógica "arriba-abajo" en el diseño y generación de muchos programas actuales, donde objetivos y necesidades son definidos desde las políticas públicas, lo que dificulta que la comunidad participe activamente en las distintas fases de los proyectos. En otras palabras, nuestros informantes censuran que las necesidades de la comunidad sean determinadas desde la voluntad política y, por lo tanto, no sean las comunidades quienes deciden si requieren o no la intervención, existiendo, además, una escasa probabilidad de rechazarla, aun cuando sus miembros evalúen que el proyecto no les favorece.

En relación con la naturaleza de los programas, nuestros entrevistados y entrevistadas denuncian la prevalencia de intervenciones enfocadas en resolver problemas que afectan a las poblaciones vulnerables, con estrategias asistenciales y de compensación de déficits, en lugar de prácticas que promuevan el desarrollo comunitario.

Por otro lado, los informantes describen problemas éticos asociados al ejercicio del rol profesional, que se relacionan tanto con el lugar en que los psicólogos se sitúan en el espacio social como con competencias profesionales específicas. Tal lugar es significado por nuestros entrevistados, metafóricamente, como el de "bisagra", en tanto se ven obligados a mediar entre las exigencias de sus financistas y las demandas de la comunidad, no siempre coincidentes entre sí. Esto se constituye en una fuente de conflictos éticos, pues sienten que deben responder a requerimientos institucionales y normativos que demandan la realización de gestiones antes de que la comunidad misma esté preparada para estas. Por ejemplo, nuestros entrevistados mencionan explícitamente un tema que afecta a los funcionarios públicos: la ley les exige denunciar delitos ante las autoridades, particularmente en casos de abuso sexual infantil (esta exigencia no rige para los psicólogos que trabajan en el sector privado). Algunos profesionales consideran que ello puede ser perjudicial para la víctima si, previo a la denuncia, no se toman ciertos resguardos en el abordaje de la situación a nivel familiar, escolar y comunitario. Otro ejemplo dice relación con los criterios de inclusión de los participantes en ciertos programas, pues hay jóvenes que, sin tener problemas legales, requieren y solicitan realizar un trabajo de promoción y bienestar, pero no son "objeto" de intervención pues no han cometido infracciones a la ley. Los profesionales perciben que es necesario buscar estrategias que permitan flexibilizar los requerimientos programáticos y adecuarlos a las necesidades de la comunidad.

Los psicólogos refieren, también, como éticamente problemática la conciliación de valores personales con valores institucionales. Cuando no son coincidentes o son discrepantes, algunos incluso han optado por renunciar a su trabajo, asumiendo los costos personales que ello implica. 
Por otra parte, los estudiantes y agentes comunitarios refieren que los miembros de la comunidad tienen elevadas expectativas respecto del rol del psicólogo. Indican que confunden sus funciones con labores de trabajo social — mal entendido-, esperando asistencia para obtener beneficios sociales a nivel municipal (e.g., subsidios, lentes). Además, nos indican que se les confunde con psicólogos clínicos, solicitándoles atención psicoterapéutica. Así, también, los usuarios de programas sociales entrevistados en este estudio esperan que el psicólogo esté siempre disponible para escuchar y contener. Ello, según estudiantes y psicólogos, ocasiona una sobredemanda hacia el profesional, incómodamente "atrapado" en una lógica asistencial de permanente ayuda e incondicionalidad. Asimismo, el problema se agudiza cuando aparece el riesgo de un quiebre en la relación con la comunidad, si no se cumplen las expectativas de los usuarios de obtener ayuda desde un rol clínico.

Respecto de la competencia profesional, tanto psicólogos como estudiantes denuncian como un problema ético la falta de experticia de otros profesionales que operan en el ámbito comunitario, señalando que se trataría de una problemática que afecta a la PC, no como una autocrítica respecto de la propia formación, sino que atribuyéndola a otros.

En cuanto a la noción de PC, los profesionales critican una falta de claridad en la utilización del concepto y límites de la disciplina cuando se denomina comunitarias a intervenciones grupales, sin importar el objetivo de la intervención ni la calidad de la participación de las personas involucradas.

Temas éticos de la formación. De la revisión de las mallas curriculares y de programas de cursos de PC y de asignaturas afines de pre y postgrado se desprende que no existe acuerdo en el modo de inclusión de la ética en su enseñanza. La formación en este ámbito se imparte -indistintamente - en diferentes niveles de las carreras de psicología y, salvo excepciones, no constituye un área de especialidad profesional al egreso de la instrucción universitaria.

Los principales problemas éticos derivan de los déficits en la formación. Los estudiantes de pre y postgrado, así como profesionales psicólogos, perciben la necesidad de formación en ética específica a la PC, pues manifiestan que en las asignaturas cursadas hubo la enunciación de temas con posibles connotaciones éticas (por ejemplo, la importancia de considerar la voz de la comunidad), pero refieren la ausencia de profundización en las modalidades y estrategias que serán abordadas en la praxis. Los estudiantes de pre y postgrado reclaman un mayor desarrollo teórico y práctico de los aspectos éticos propios del quehacer comunitario, al considerar que la formación teórica por sí sola es insuficiente para decantar la aplicabilidad de los contenidos sin incurrir en faltas éticas. Perciben una falta de herramientas que les permita identificar y resolver problemas éticos en esta área disciplinar, particularmente por la ausencia en la formación de casos prácticos o ejemplos de cómo abordar problemas concretos y reales.

Los estudiantes también cuestionan el inicio abrupto de trabajos prácticos en terreno sin la supervisión adecuada, lo que, en consecuencia, les dificulta conciliar adecuadamente las demandas de la comunidad con las institucionales y los requerimientos académicos.

En respuesta a tales déficits, apreciamos un verdadero anhelo por experiencias formativas que incluyan el análisis y discusión de situaciones propias del ejercicio comunitario, así como, también, por la formación en competencias éticas específicas.

Entre los problemas derivados de los requerimientos de las instituciones donde los estudiantes realizan su práctica profesional, destaca la solicitud de desempeñar funciones clínicas, ajenas a la PC, así como la exigencia de hacerse cargo de proyectos e intervenciones para las que no están preparados y no contar con la supervisión requerida en terreno y/o en la universidad.

Entre los recursos, los estudiantes valoran los aportes de Maritza Montero en cuanto a hacer visible la dimensión ética, aunque reconocen la escasa formación recibida en el tema. 
Temas éticos en investigación en PC. Un primer resultado es la escasa presencia de los temas éticos en la investigación comunitaria, evidente tanto en las entrevistas individuales y grupales y el grupo Delphi como en los documentos analizados. Nuestros expertos prácticamente no anticipan desafíos éticos particulares para la investigación en PC. La revisión de códigos deontológicos y pautas éticas orientadoras no entrega normativa particular para la investigación en PC.

Sin embargo, a partir del meta-análisis de tesis para obtener el título de psicólogo o el grado de magíster en PC, identificamos temas éticos propios del proceso de producción de conocimiento científico en este ámbito disciplinar, categorizados en cuatro grupos que describimos a continuación.

En primer lugar, en las tesis revisadas se aprecia, implícita o explícitamente, una preocupación por aspectos éticos, como el manejo confidencial de la información y la obtención de consentimiento informado de parte de los sujetos de investigación. En una proporción muy menor encontramos declaraciones respecto de la dimensión ética y política de la investigación comunitaria. La inclusión de un apartado intitulado aspectos éticos o consideraciones éticas aparece en algunas tesis de postgrado y solo en una de pregrado.

Respecto de problemas éticos en la relación con los participantes de la investigación, encontramos que el respeto del anonimato en la publicación de nombres y apellidos de participantes, así como del nombre de la institución donde se realizó la investigación, es transgredido con cierta frecuencia.

Dificultades para acreditar el carácter voluntario de la participación de las personas en los estudios aparecen al omitir, en las tesis, información referente a permisos o la obtención de consentimiento informado por parte de la comunidad o enunciando el proceso de consentimiento informado muy tangencialmente. Ello también ocurre en el caso de la participación de menores de edad, pues en ninguna de las tesis en que los sujetos eran jóvenes y niños encontramos referencia a la solicitud de su asentimiento, aunque en ocasiones se mencione la solicitud de consentimiento informado a padres y apoderados. Observamos, también, el uso de material audiovisual, fotos y grabaciones en video, sin solicitar autorización especial a las personas que aparecen en ellos, negando su carácter de información sensible, protegida incluso por ley, y develando la identidad de la persona en situaciones que pueden ser comprometedoras o generar conflictos entre los miembros de la comunidad.

Por otro lado, apreciamos un cierto descuido en los usos semánticos, al utilizar en títulos y textos vocablos que promueven un menoscabo de la imagen de los participantes, con el consiguiente riesgo de estigmatización. Paradigma de tal cuestión es la referencia a "drogadictos" o "esquizofrénicos".

Respecto del diseño de la investigación, encontramos que no hay devolución de resultados a la comunidad, postura que privilegia los beneficios que supuestamente la investigación producirá en el avance del conocimiento, negando a la comunidad el acceso a su propia información y a los posibles efectos positivos que de ella podrían derivarse.

$\mathrm{Y}$ en cuanto a problemas derivados de la recolección de datos, las investigaciones que analizamos utilizaron preferentemente métodos cualitativos para la recolección y análisis de la información, siendo la técnica más utilizada la entrevista, en sus diversas modalidades, tanto individual como grupal. Un cuestionamiento ético nos merece el encuadre inapropiado para la realización de entrevistas, por ejemplo, efectuarlas en presencia de terceros, clientes o colegas, lo que no garantiza un espacio protegido para la obtención de información e, incluso, amenaza la calidad de la información obtenida y con ello el valor científico de la investigación. 


\section{Orientaciones Éticas Específicas Para la PC}

Como resultado de la revisión documental, detectamos principios y normas éticas aplicables a la PC, particularmente en los códigos deontológicos de Canadá (Canadian Psychological Association, 2000) y en el recientemente aprobado Código de Guatemala (Angelique \& Kyle, 2002), única propuesta deontológica específica para la PC.

Considerando particularmente las normas de tales códigos y los resultados obtenidos en esta investigación, iniciamos un proceso reflexivo como equipo, incluyendo también nuestra propia experiencia como psicólogas comunitarias, docentes e investigadoras. Como producto de tal proceso, proponemos una lista de deberes éticos para el quehacer profesional. Sus contenidos pertenecen a los participantes de nuestra investigación, pero su formulación y redacción es de nuestra responsabilidad, tal como aparecen en la Tabla 3.

Nos parece que esta lista de deberes constituye un marco de referencia explícito para el quehacer profesional de psicólogos comunitarios en distintos campos de acción: académico, en terreno y en su intersección. Sin embargo, como ética mínima, siguiendo a Cortina (2008), en ningún caso es exhaustiva ni agota los aspectos que se deben tener en cuenta en el ejercicio profesional. Excluimos de este trabajo el análisis de la pertinencia de tales deberes a otras disciplinas.

Será tarea de las sociedades científicas y asociaciones gremiales decidir si adoptan estas orientaciones éticas como recomendación o exigencia para el quehacer de sus asociados.

Tabla 3

Deberes Éticos en PC

$>$ Deber de conocer y respetar el sistema de valores y las estructuras sociales que operan en la comunidad

$>$ Deber de explicitar las condiciones de inserción en la comunidad

$>$ Deber de promover la participación comunitaria en todas las etapas de la intervención

$>$ Deber de informar a la comunidad todos los aspectos vinculados a la intervención, privilegiando los que contribuyan a la resolución de sus problemas

$>$ Deber de reconocer y valorar otros saberes, de otros profesionales y de miembros de la comunidad

$>$ Deber docente de reconocer el carácter ético y político de la PC como ciencia y como profesión

$>$ Deber de enfatizar aspectos éticos propios de la PC

$>$ Deber de impartir una formación teórico-práctica

$>$ Deber de supervisar las prácticas estudiantiles

$>$ Deber de informar a la comunidad cuando se está llevando a cabo una investigación científica

$>$ Deber de conocer y respetar el sistema de valores y las estructuras sociales comunitarias, integrándolas en el diseño de la investigación

$>$ Deber de obtener consentimiento libre e informado de los participantes y representantes de la comunidad, cuando corresponda

$>$ Deber de considerar resguardos especiales en el registro y divulgación de información por medio de imágenes, registros audiovisuales y formatos digitales

$>$ Deber de promover formas participativas de investigación

Deber de difundir los resultados de la investigación a la propia comunidad

$>$ Deber de considerar la coautoría de la comunidad en la publicación de los resultados de la investigaciones 


\section{Discusión y Conclusiones}

Respecto de la práctica en PC, los resultados refuerzan la advertencia ya realizada por otros autores (Alfaro, 2007; Alfaro \& Zambrano, 2009; Asún, 2007; Krause, 2002; Krause \& Jaramillo, 1998; Winkler et al., 2010) al problematizar los alcances de la institucionalización de esta disciplina en los últimos años. Considerando los testimonios de nuestros entrevistados, observamos que la incorporación de las prácticas comunitarias en programas y políticas públicas tiene como efecto, por una parte, la ventaja de una mayor inserción profesional, pero, por otra, se constituye en una de las principales fuentes de tensiones éticas experimentadas por los profesionales del área.

Existe consenso respecto de las interferencias que los programas y políticas diseñados "desde arriba" generan en las prácticas comunitarias actuales, por lo que se trata de una condición de contexto que debe ser considerada para pensar el lugar de la ética en el quehacer comunitario y para repensar los fundamentos del enfoque. Consideramos que, desde el punto de vista ético y rescatando el aporte de Prilleltensky (2001), sería relevante estudiar, junto con legisladores y operadores políticos, modos en que se articulen consistentemente políticas, programas y objetivos con los valores éticos que promueve la PC.

Considerando, entonces, la determinación que el contexto — dado por las políticas públicasejerce sobre la práctica comunitaria, creemos necesario impulsar estrategias que permitan permear a los actores que participan en la génesis y diseño de los programas. Coincidimos, en este sentido, con las reflexiones de Alfaro y Zambrano (2009), respecto de la urgencia de generar puentes de interlocución y superar las limitaciones disciplinares que circunscriben el debate a un ámbito específico. Resulta importante reconocer que las políticas y programas se diseñan e implementan luego de sucesivos procesos de presión, alianzas y negociaciones, en los que las posiciones de los actores involucrados dependerán tanto de las lógicas que dan cuerpo a una política como del lugar y visibilidad técnica que esta alcance. En este sentido, constituye una limitación de nuestro trabajo la no inclusión de diseñadores de políticas públicas y de programas psicosociales como fuentes de información, tarea pendiente para futuros estudios.

Reconocemos que para establecer diálogos fructíferos con quienes toman decisiones, financian y formulan políticas es indispensable fortalecer la identidad disciplinar y consolidar a la PC como una especialidad reconocida y valorada, tanto en la comunidad científica y profesional como en la sociedad global, particularmente en su capacidad de entregar respuestas eficientes a los problemas psicosociales que afectan a las comunidades. Para ello es fundamental comprometer nuestras voluntades en convocar a nuestros pares y aunar esfuerzos para la consolidación de la disciplina, a través de acciones gremiales, científicas y políticas. Pero, también, importa ser conscientes de la tarea pendiente de formalizar la PC como especialidad en Chile. Ello implica promover mecanismos de acreditación que garanticen una formación específica y pertinente que proporcione, también desde lo técnico, herramientas para identificar y resolver problemas éticos y, así, asegurar que los destinatarios de las intervenciones implementadas puedan participar y decidir respecto de los problemas que les afectan y de sus soluciones.

Frecuentes tensiones éticas se relacionan con las dificultades que tanto estudiantes como profesionales presentan al momento de establecer los límites de su quehacer. Tales dificultades pueden vincularse con una conceptualización difusa y poca claridad respecto de las prácticas propias de la disciplina, la connotación ideológica atenuada y el escaso entrenamiento en habilidades, competencias y contenidos específicos para este campo. Estas falencias contribuyen a que el lugar del otro pierda nitidez en el enfoque comunitario. Así, entonces, la comunidad y su participación parecieran quedar relegadas a los márgenes de las intervenciones, a pesar de ser el principio aspiracional más evocado por nuestros entrevistados al momento de reflexionar sobre la ética.

También preocupa que tanto los profesionales como los expertos del panel Delphi visualicen la comunidad desde la carencia, esquivando en sus intervenciones las apuestas más promocio- 
nales y transformadoras. Ello nos muestra un serio problema, pues la PC surge como una disciplina que reconoce las capacidades y recursos que los grupos y comunidades han ido adquiriendo en sus distintos trayectos. La realidad del quehacer comunitario que muestran los resultados obtenidos en nuestro estudio no refleja, e incluso contradice, tanto los planteamientos teóricos propios de la PC como la valoración de la participación y desarrollo de empowerment y fortalecimiento que promueven, por ejemplo, Rappaport (1981), Ortiz (1998) y Montero (2006).

Los resultados retratan un panorama complejo e inhóspito para los psicólogos comunitarios, debido a que, por una parte, no hay consenso respecto a cómo enseñar PC y la formación que se entrega en el área no constituye una especialidad profesional al egreso universitario y, por otra parte, hay ausencia de normativas que regulen el ejercicio profesional, existiendo un desconocimiento de lo esperado para el rol en usuarios, legisladores y operadores de las políticas públicas, estudiantes de psicología e, incluso, entre los mismos psicólogos. De acuerdo a la propuesta de Pasmanik y Winkler (2009), el ciclo profesional sería el momento para conocer en detalle el código de ética profesional y las normas legales asociadas al ejercicio profesional y, agregamos ahora, las normativas específicas para la PC.

Un gran desafío ético para los psicólogos comunitarios en Chile es contribuir, como ya mencionamos, al reconocimiento, acreditación y regulación profesional de la PC. Un avance es la formación en ciernes de la Sociedad de Psicólogos Comunitarios en el año 2009 en la ciudad de Temuco.

Cuando nuestros entrevistados denuncian los problemas éticos que identifican para la PC muestran que están impregnados por los valores que la inspiran y que promueve, pero al momento de la praxis la actualización de tales valores se ve obstaculizada, en parte por las limitaciones contextuales descritas previamente.

Los problemas éticos en la investigación comunitaria pueden ser resumidos en las dificultades para considerar a las personas e incluir la participación de la comunidad en el proceso. Pareciera que, tal como ocurre en la práctica en PC, en la investigación tampoco se consideran las necesidades de la comunidad en la generación de conocimiento o en la producción de un saber que le pueda ser de utilidad. En demasiados casos se tiende a estudiar a las personas como "objetos", obturando su participación como sujetos productores de conocimiento válido y con el derecho a que se respete su dignidad. Otros problemas, tales como acreditar el carácter voluntario de la participación de los sujetos en la investigación, han sido consignados no solo para la PC sino para todas las ciencias sociales (Lira, 2008).

Montero (2004a) plantea que una pregunta que siempre debemos formular al momento de iniciar un estudio se relaciona con la necesidad de declarar explícitamente si aquello que se hace está o no libre de valores y, en este sentido, desde la perspectiva de la PC, se espera que quienes hagan una investigación expresen sus sesgos, sus tendencias, sus creencias y compromisos con posiciones, no solo teóricas, sino también políticas y religiosas. Asimismo, Montero agrega que muchos de estos sesgos se vinculan con los compromisos morales que se han adquirido a lo largo de la vida y, por tanto, determinarán el desarrollo de la investigación en toda su amplitud, incluso en la elección misma del tema de investigación. En este estudio en particular no escapamos de estos sesgos y el problema no está en que existan, sino en el riesgo de ocultarlos. El sesgo a la base de esta investigación es que la PC debe ser coherente y consecuente con los valores que inspiraron su desarrollo en Latinoamérica y sus respectivas exigencias éticas. Los resultados muestran que el contexto actual dificulta desempeñarnos éticamente en el ámbito comunitario, pues las políticas públicas que enmarcan el quehacer nos obligan a anteponer la técnica por sobre la ética, transformándonos en operadores que ejecutan lo dispuesto por otros.

Concluimos que querer que la comunidad participe, que los programas tengan objetivos transformadores o respetar los valores propios de la comunidad, en su dimensión eminentemente aspiracional, parece insuficiente, más aún cuando al contexto descrito se suma la preca- 
ria formación y la falta de pautas orientadoras y de normativas reguladoras para un ejercicio profesional éticamente impecable. Con nuestra propuesta de una lista de deberes éticos para los psicólogos comunitarios esperamos contribuir a disminuir la distancia entre los ideales aspiracionales y la práctica.

\section{Referencias}

Alfaro, J. (2007). Políticas sociales como condición de posibilidad para el desarrollo de prácticas en psicología comunitaria. En J. Alfaro \& H. Berroeta (Eds.), Trayectoria de la psicología comunitaria en Chile: prácticas y conceptos (pp. 43-72). Valparaíso, Chile: Universidad de Valparaíso.

Alfaro, J. \& Zambrano, A. (2009). Psicología comunitaria y políticas sociales en Chile. Psicologia \& Sociedade, 21, $275-282$

Álvarez, J. (2000). Investigación cuantitativa/investigación cualitativa: ¿una falsa disyuntiva? En T. D. Cook \& C. S. Reichardt (Comps.), Métodos cualitativos y cuantitativos en investigación evaluativa (pp. 9-24). Madrid, España: Morata.

Angelique, H. \& Kyle, K. (Redact.) (2002). Monterey Declaration of Critical Community Psychology (adoptada en la Inaugural Critical Psychology Conference, Monterey, CA, Estados Unidos, Abril 2001). The Community Psychologist, 35(1), 35-36.

Asún, D. (2007). Entrevista Domingo Asún. En J. Alfaro \& H. Berroeta (Eds.), Trayectoria de la psicología comunitaria en Chile: prácticas y conceptos (pp. 413-432). Valparaíso, Chile: Universidad de Valparaíso.

Banyard, V. L. \& Miller, K. E. (1998). The powerful potential of qualitative research for community psychology. American Journal of Community Psychology, 26, 485-505. doi:10.1023/A:1022136821013

Berdullas, M., Santolaya, F. \& Fernández, J. R. (2002). La década 1989-1998 en la psicología española: análisis del desarrollo de la psicología profesional en España. Papeles del Psicólogo, 82, 65-82.

Bersoff, D. N. (1995). Ethical conflicts in psychology. Washington, DC: American Psychological Association.

Bolívar, A. (2005). El lugar de la ética profesional en la formación universitaria. Revista Mexicana de Investigación Educativa, 10(24), 93-123.

Canadian Psychological Association (2000). Canadian code of ethics for psychologists. Québec, Canadá: Autor.

Castillo, J. \& Winkler, M. I. (2010). Praxis y ética en psicología comunitaria: representaciones sociales de usuarias y usuarios de programas comunitarios en la Región Metropolitana. Psykhe, 19(1), 31-46. doi:10.4067/S071822282010000100003

Cortina, A. (2008). Ética mínima: introducción a la filosofía práctica. Madrid, España: Tecnos.

Cushman, P. (1993). Psychotherapy to 1992: A historically situated interpretation. En D. K. Freedheim, H. J. Freudenberger, J. W. Kessler, S. B. Messer, D. R. Peterson, H. H. Strupp \& P. L. Wachtel (Eds.), History of psychotherapy: A century of change (pp. 21-64). Washington, DC: American Psychological Association. doi:10.1037/10110-000

Erikson, K. T. (1967). A comment on disguised observation in sociology. Social Problems, 14, 366-373.

Flick, U. (1990). Fallanalysen: Geltungbegründung durch systematische Perspektiven-Triangulation [Análisis de casos: fundamentación de la calidad a través de la triangulación sistemática de perspectivas]. En G. Jüttemann (Ed.), Komparative Kasuistik [Casuística comparativa] (pp. 184-203). Heidelberg, Alemania: Asanger.

González, F. (1998). Epistemología y subjetividad. Revista Interamericana de Psicología, 32,139-167.

González, M. (2002). Aspectos éticos de la investigación cualitativa. Revista Iberoamericana de Educación, 29, 85-103. Extraído de http://www.campusoei.org/salactsi/mgonzlez5.htm

Guatemala, Colegio de Psicólogos (2010). Código de ética. Ciudad de Guatemala: Tribunal de Honor.

Heller, K. (1989). The return to community. American Journal of Community Psychology, 17, 1-15. doi:10.1007/ BF00931199

Hombrados, M. I. (1996). Introducción a la psicología comunitaria. Málaga, España: Aljibe.

Jenkins, R. A. (2010). Applied roles and the future of community psychology. American Journal of Community Psychology, 45, 68-72. doi:10.1007/s10464-009-9285-1

Jimeno, P. (2011, Abril 11). Chile transparentará por primera vez gastos e impacto de programas sociales. La Tercera, Negocios, p. 19.

Krause, M. (2002). The institutionalization of community interventions in Chile: Characteristics and contradictions. American Journal of Community Psychology, 30, 547-570. doi:10.1023/A:1015812118935

Krause, M. \& Jaramillo, A. (1998). Intervenciones psicológico-comunitarias en Santiago de Chile. Santiago, Chile: Pontificia Universidad Católica de Chile.

Levine, M., Perkins, D. D. \& Perkins, D. V. (Eds.) (1997). Principles of community psychology: Perspectives and applications (2a ed.). New York, NY: Oxford University Press.

Lira, E. (Ed.) (2008). Bioética en investigación en ciencias sociales. Santiago, Chile: Comisión Nacional de Investigación Científica y Tecnológica.

Marconi, J. (1986). Política de salud mental en América Latina (reimpresión). Revista Chilena de Psicología, 8(2), 3339.

Martínez, M. (1999). Criterios para la superación del debate metodológico "cuantitativo/cualitativo". Revista Interamericana de Psicología, 33, 79-107.

Montero, M. (1984). La psicología comunitaria: orígenes, principios y fundamentos teóricos. Revista Latinoamericana 
de Psicología, 16, 387-400.

Montero, M. (2004a). Introducción a la psicología comunitaria. Buenos Aires, Argentina: Paidós.

Montero, M. (2004b). Relaciones entre psicología social comunitaria, psicología crítica y psicología de la liberación: una respuesta latinoamericana. Psykhe, 13(2), 17-28. doi:10.4067/S0718-22282004000200002

Montero, M. (2006). Hacer para transformar: el método en la psicología comunitaria. Buenos Aires, Argentina: Paidós.

Montero, M. \& Winkler, M. I. (2012). Psicología comunitaria, ética y futuro: una prospección realizada por personas expertas. Manuscrito en preparación.

O'Neill, P. (1989). Responsible to whom? Responsible for what? Some ethical issues in community intervention. American Journal of Community Psychology, 17, 323-341. doi:10.1007/BF00931040

Orellana, M. (1994). Pluralismo: una ética para el siglo XXI. Santiago, Chile: Editorial Universidad de Santiago.

Ortiz, B. (1998). El empowerment como alternativa teórica para la psicología de comunidad en América Latina. Revista Interamericana de Psicología, 33, 49-66.

Pasmanik, D. \& Winkler, M. I. (2009). Buscando orientaciones: pautas para la enseñanza de la ética profesional en psicología en un contexto con impronta postmoderna. Psykhe, 18(2), 37-49. doi:10.4067/S071822282009000200003

Polit, D. \& Hungler, B. (2002). Investigación científica en ciencias de la salud. México, DF: McGraw-Hill.

Pope, K. S. (1990). Identifying and implementing ethical standards for primary prevention. En G. B. Levin, E. J. Trickett \& R. E. Hess (Comps.), Ethical implications of primary prevention (pp. 43-64). New York, NY: Haworth Press.

Pope, K. S. (1991). Dual relationships in psychotherapy. Ethics \& Behavior, 1, 21-34. doi:10.1207/s15327019eb0101_3

Prilleltensky, I. (2001). Value-based praxis in community psychology: Moving toward social justice and social action. American Journal of Community Psychology, 29, 747-778. doi:10.1023/A:1010417201918

Rappaport, J. (1976). Community psychology: Values, research and action. New York, NY: Holt, Rinehart and Winston.

Rappaport, J. (1981). In praise of paradox: A social policy of empowerment over prevention. American Journal of Community Psychology, 9, 1-25. doi:10.1007/BF00896357

Rappaport, J., Swift, C. \& Hess, R. (1984). Studies in empowerment: Steps toward understanding and action. New York, NY: Haworth Press.

Reyes, M. I. (2007). Orientaciones ético-valóricas de la psicología comunitaria en Chile: análisis descriptivo de los artículos publicados en revistas nacionales entre 1993 y 2003. En J. Alfaro \& H. Berroeta (Eds.), Trayectoria de la psicología comunitaria en Chile: prácticas y conceptos (pp. 109-141). Valparaíso, Chile: Universidad de Valparaíso.

Reyes, M. I. (2009). Representaciones sociales del ejercicio y la conducta ética profesional del/la psicólogo/a en dos medios de comunicación de masas (Tesis de Magíster no publicada), Escuela de Psicología, Universidad de Santiago de Chile.

Sánchez Vidal, A. (1999). Ética de la intervención social. Buenos Aires, Argentina: Paidós.

Serrano-García, I. \& Vargas, R. (1993). La psicología comunitaria en América Latina: estado actual, controversias y nuevos derroteros. Papeles del Psicólogo, 55, 41-47.

Silva, C. \& Martínez, M. (2007). Empoderamiento: proceso, nivel y contexto. En J. Alfaro \& H. Berroeta (Eds.), Trayectoria de la psicología comunitaria en Chile: prácticas y conceptos (pp. 305-322). Valparaíso, Chile: Universidad de Valparaíso.

Stein, C. H. \& Mankowski, E. S. (2004). Asking, witnessing, interpreting, knowing: Conducting qualitative research in community psychology. American Journal of Community Psychology, 33, 21-35. doi:10.1023/B: AJCP.0000014316.27091.e8

Wiesenfeld, E. (2000). Entre la prescripción y la acción: la brecha entre la teoría y la práctica en las investigaciones cualitativas. FQS Forum: Qualitative Social Research, 1(2), artículo 30. Extraído de http://www.qualitativeresearch.net/fqs-texte/2-00/2-00wiesenfeld-s.htm

Winkler, M. I. (1999). Los derechos de los pacientes o clientes: relaciones duales y conducta sexual inapropiada. Terapia Psicológica, 17, 201-206.

Winkler, M. I. (2007). Cuestiones éticas en psicología comunitaria: dudas en la praxis. En J. Alfaro \& H. Berroeta (Eds.), Trayectoria de la psicología comunitaria en Chile: prácticas y conceptos (pp. 373-400). Valparaíso, Chile: Universidad de Valparaíso.

Winkler, M. I., Alvear, K., Olivares, B. \& Pasmanik, D. (2010). Autonomía versus dependencia: quehacer comunitario y políticas públicas. Santiago, Chile: Universidad de Santiago de Chile.

Winkler, M. I., Alvear, K., Olivares, B. \& Pasmanik, D. (en prensa). Lo ético es transversal y cotidiano: dimensiones éticas en la formación y práctica en psicología comunitaria. Actas Bioethica.

Winkler, M. I. \& Reyes, M. I. (2006). Representaciones sociales de psicólogos chilenos acerca del ejercicio profesional ético. Fundamentos en Humanidades, 13-14, 63-89.

Fecha de recepción: Marzo de 2011.

Fecha de aceptación: Enero de 2012. 